\title{
Application of Intratissue
}

\section{Diadynamophoresis of Antibiotics}

and Ozonized Saline Solution in

Multimodality Treatment of

Congenital Hydronephrosis in

\section{Children}

\author{
Maher Yousef, MD, PhD* \\ Vyacheslav Davidenko, MD, PhD* \\ Syed Hasan, MD, FABHP ${ }^{\dagger}$ \\ Mahmoud Yousef, $\mathrm{PhD}^{\ddagger}$
}

\author{
*Kharkov State Medical University, Kharkiv, Ukraine \\ ${ }^{\dagger}$ Western Missouri Medical Center, Warrensburg, Missouri \\ ${ }^{\ddagger}$ Central Missouri State University, Warrensburg, Missouri
}

KEY WORDS: diadynamophoresis, congenital hydronephrosis, pyelonephritis, Andersen-Hynes operation

\section{ABSTRACT}

Objective: Malformations of the urinary system (US) are among the most widespread anomalies of development.

Congenital hydronephrosis, typically caused by pyeloureteral segment obstruction, is a frequent pathology in children and is often complicated by pyelonephritis. The purpose of this study is to improve the results of treatment of congenital hydronephrosis complicated by obstructive pyelonephritis in children by means of early surgical correction of the malformation that causes hydronephrosis and to increase the efficiency of antibacterial therapy through applying intratissue diadynamophoresis of antibiotics and urine sanitation with ozonized solutions.
Methods: The study was conducted in two parts: an experimental section and clinical research. In the experimental section four series of tests were carried out on young Chinchilla rabbits ( 72 experiments). The first two series of tests were devoted to the study of gentamicin concentration in renal parenchyma and urine with different methods of antibiotic administration. The second experiment on animals studied the concentration ability of diadynamophoresis of antibiotics. The clinical research consisted of studying 125 children with congenital hydronephrosis caused by obstructive anomalies of pelviureteral segment and complicated by secondary pyelonephritis. The children were also divided into two groups. The first group (control, $\mathrm{n}=50$ ) received a standard regimen of post-operative antibacterial therapy while the second group (main, $n=75$ ) underwent intratissue diadynamophoresis. 
Results: Our study shows the advantage of early surgical correction of congenital hydronephrosis for preventing renal parenchyma atrophy, nephrosclerosis development, and urinary tract infection. For treating the secondary pyelonephritis, the application of intratissue diadynamophoresis of antibiotics with a novel device showed statistically significant improvement in patient recovery. For the first time this research uses renal excretory system and urine sanitation with ozonized saline solution.

Conclusion: Early surgical correction of malformations and anomalies of urinary system development enables timely restoration of normal urodynamics and prevention of further hydronephrotic transformation of kidney, as well as development of secondary pyelonephritis. The developed methods of integrated treatment of secondary pyelonephritis are highly effective, economical and accessible.

\section{INTRODUCTION}

In recent years, the range of options for the surgical treatment of hydronephrosis in children has been greatly expanded. Among those aimed at the restoration of urodynamics, the most frequently used is the Anderson-Hynes operation which involves the resection of pathologically formed pyeloureteral segment and creation of anastomosis between the pelvis and ureter. However, the results of therapy for this pathology depend not only on the method of operation, but also on such important aspects as the initial state of the kidney and the absence or presence of inflammation in the parenchyma and urinary tract.

The main cause of pyelonephritis development in children is congenital anomalies and malformations in the form of urinary system obstructions resulting in impairment of urodynamics, urine stagnation, and infections. ${ }^{1}$
Contemporary microflora, causing secondary pyelonephritis, is characterized with considerable variety, high virulence, and resistance.

Long-term existence of hydronephrosis, complicated with secondary pyelonephritis, leads to the development of nephrosclerosis, secondary renal scarring, and renal insufficiency. The presence of infection in the urinary system can result in scarring in the area of anastomosis and stricture formation. In addition, hydronephrotic transformation accompanied with pyelonephritis and cicatricosclerotic changes in the renal parenchyma cause serious disturbances of blood circulation in the kidney. This complicates the penetration of antibacterial agents into all renal sections and significantly reduces the efficacy of pyelonephritis treatment. $^{2}$

The given peculiarities of hydronephrosis complicated with secondary pyelonephritis in children have led researchers to look for new methods of effective therapy. Despite much success, current treatment protocols for hydronephrosis in children are far from perfect, and further research in this field is needed.

The important aspect of antibacterial therapy is the concentration of antibiotics in the kidney and urinary tract. One effective method designed to provide high concentration of antibacterial agents in the inflamed area in spite of microcirculation impairments is intratissue diadynamophoresis (DDP) of antibiotics. $^{3}$ In the available literature, however, we could not find any information about the efficacy of intratissue DDP of antibiotics for treatment of hydronephrosis against a background of secondary pyelonephritis in children. Therefore, we decided to undertake this study.

One important factor in suppressing urine excretory system infection is direct urine sanitation. Recent data show high 


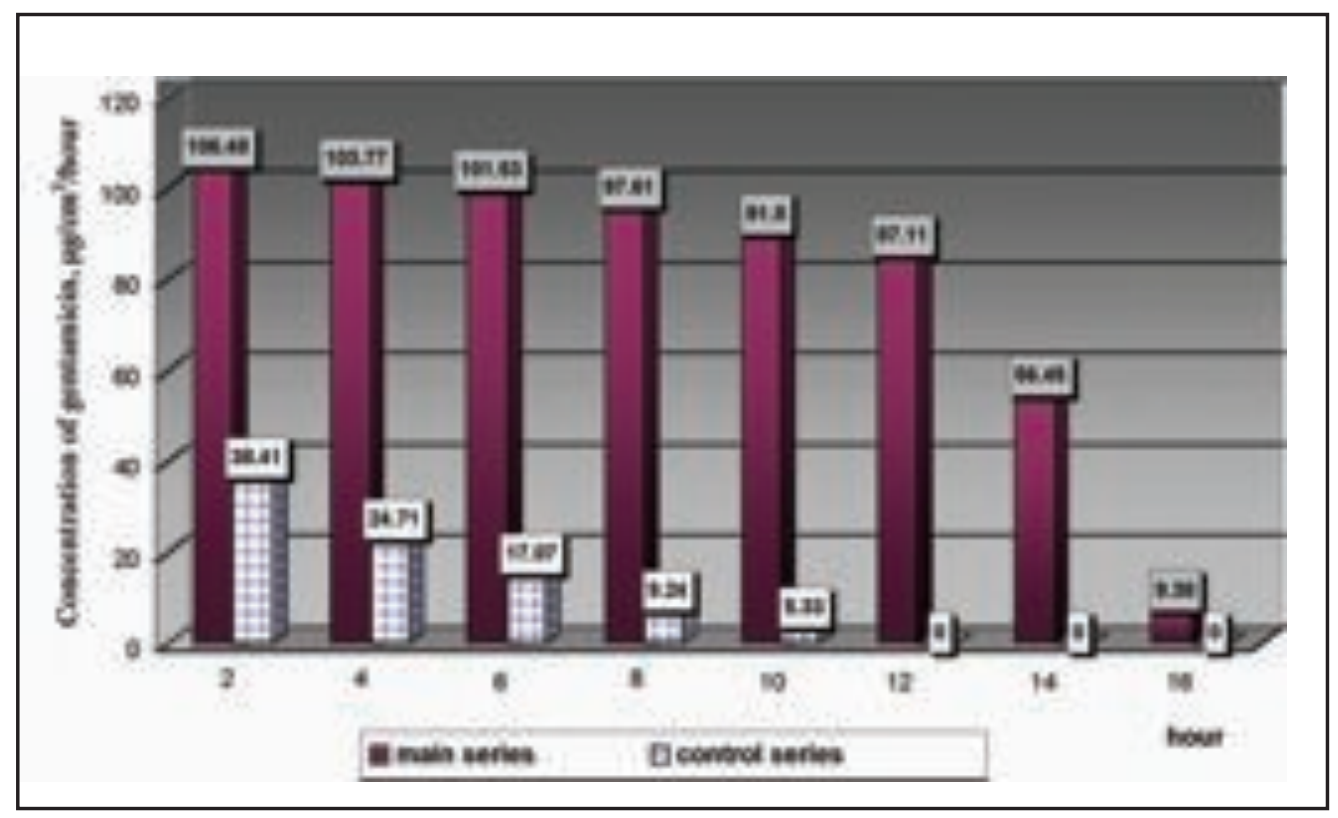

Figure 1. Dynamics of hourly difference of gentamicin concentrations in renal parenchyma.

bactericidal activity of ozonized solutions in relation to the majority of grampositive, gram-negative, and anaerobic microorganisms. ${ }^{4}$ Direct studies of the use of ozonized solutions for sanitation of children's urinary system and urine were not found. Studies of this method of treatment of secondary pyelonephritis, in our opinion, are quite relevant and promising.

\section{STUDY OBJECTIVES}

The objective of this study was to examine the incidence of secondary pyelonephritis in children with hydronephrosis in different age groups. Specifically, we planned to study species composition of children's secondary pyelonephritis microflora and its sensitivity to antibiotics and to develop experimentally and introduce to medical practice the method and device for DDP of antibiotics at secondary pyelonephritis in children. We also planned to study the efficiency of renal excretory system and urine sanitation with ozonized saline solution, and assess the efficiency of treatment of children with congenital hydronephrosis complicated with secondary pyelonephritis by studying immediate and long-term results.

\section{MATERIALS AND METHODS} Animal Experiments

The study consisted of two parts: an experimental section and clinical research. The experimental part of this research was devoted to the study of the concentration ability of DDP of antibiotics. For this purpose, four series of tests on young Chinchilla rabbits ( 72 experiments) were carried out.

The first two series of tests were devoted to the comparative study of gentamicin concentration in renal parenchyma and urine with different methods of antibiotic administration. For this, the ear edge vein and urinary bladder were catheterized in animals of series one (control, 20 animals) and gentamicin, 5 $\mathrm{mg} / \mathrm{kg}$, was administered by intravenous (IV) drops within 30 minutes.

To determine the gentamicin concentration in renal parenchyma, $500 \mathrm{mg}$ of renal tissue were obtained. Study materials were obtained at 2-hour inter- 


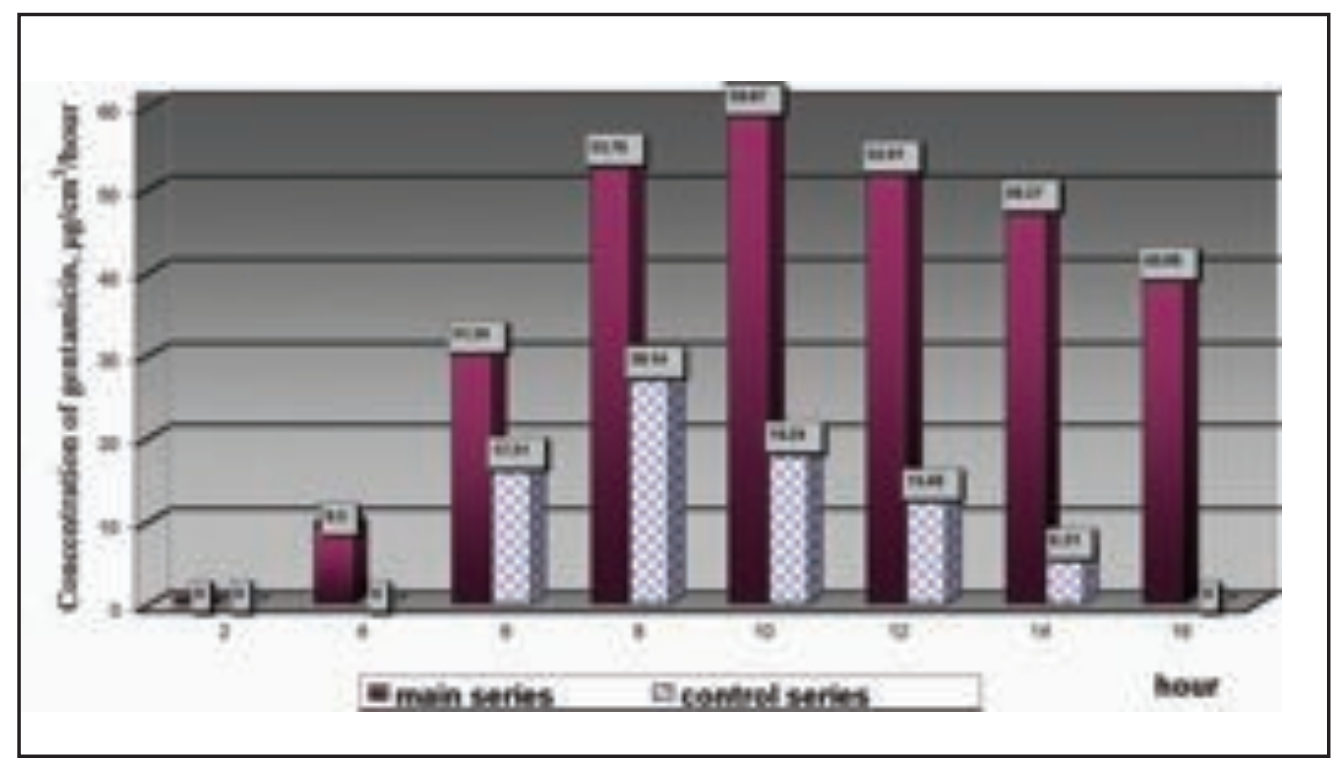

Figure 2. Dynamics of hourly difference of gentamicin concentrations in the urine.

vals beginning at hour 2 and ending at hour 16. Urine was taken at the same time. Determination of gentamicin concentration in biological substrates was carried out by the method of agar diffusion effected in two layers of medium on Petri dish.

The second experiment on animals (main, 32 animals) was carried out using the following methods: after removing all of the hair from the abdomen and back under IV hexenalum anesthesia, lumbotomy was performed, the kidney was mobilized from the surrounding tissues, the pelvis was opened, and a microirrigator with numerous openings was introduced to the ureter through the renal parenchyma. A flexible wire electrode was inserted in the lumen with output to the back skin.

The next day a gentamicin solution $(5 \mathrm{mg} / \mathrm{kg}$ ) was administered to the ear edge vein using the drop method.

External electrodes were fixed on the anterior abdominal wall and back. All electrodes were connected to a Tonus device (Apparatus machine for treatment by Dynomic Electric stream, TONUS_1 DT-50-3, OOO Neva-Treid,
St. Petersburg, Russia). The kidney was treated with two-semiperiodic continuous diadynamic current $0.5 \mathrm{amp}$ for 15 minutes at the time of IV administration of antibiotic, and continued for $15 \mathrm{~min}$ utes after stopping the infusion.

With the aim of comparative analysis of the efficiency of intratissue DDP of antibiotics in conditions of experimental pyelonephritis, two more series of experiments were performed. In these experiments, pyelonephritis in rabbits (20 chinchilla rabbits) was induced by IV administration of day culture of plasmacoagulating staphylococcus (ATCC25923) containing 800 million microbial bodies.

Development of inflammatory process and confirmation of pyelonephritis were seen in the course of the examination of the renal preparations taken on the third and sixth day after infection. The experimental methods of the third series (10 animals) corresponded to the first series and the methods of the fourth series (10 animals) corresponded to the second series. Therapy and study of its efficacy took 10 days. Urine tests for determining the bac- 


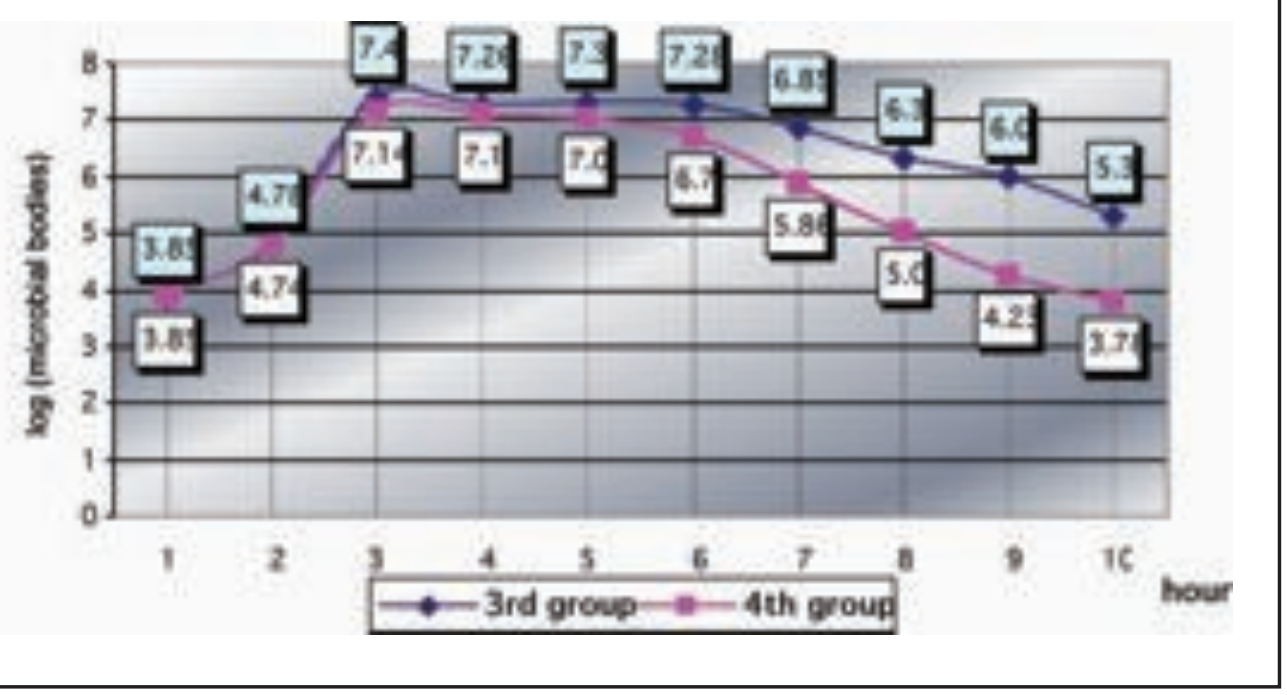

Figure 3. Dynamics of bacteriuria parameter logarithm in animals of the third and fourth series of experiment.

teruria and leukocyturia was performed daily. A histological study of morphological changes in kidneys was carried out on the third, sixth, and tenth day of the experiment.

\section{Clinical Studies}

All clinical studies were performed at the Child Surgical Clinic of Kharkiv Regional Child Clinical Hospital No.1, Ukraine. Consents were obtained from the parents of patients prior to study.

Clinical observations consisted of studying 125 children with congenital hydronephrosis caused by obstructive anomalies of pelviureteral segment and complicated by secondary pyelonephritis ( 84 boys and 41 girls). The ages of children were from birth to 14 years.

All children with congenital hydronephrosis complicated with pyelonephritis underwent complex therapy consisting of pre-operative preparation, operation, and post-operative conservative treatment. They underwent similar operations: resection of pyeloureteral segment according to Anderson-Hynes.
In order to compare the efficacy of treatment of secondary pyelonephritis, the patients were divided into two groups. The first group (controls, $\mathrm{n}=50$ ) underwent a standard regimen of postoperative antibacterial therapy. In the second group (main, $\mathrm{n}=75$ ) administration of antibiotic was performed using our methods, and pelvis and ureter were sanitized using drop-by-drop administration of ozonized saline solution (6-8 $\mathrm{mg} / \mathrm{L})$.

To perform intratissue DDP of antibiotics in clinical conditions a device composed of two electrodes, one of which was designed as a flexible plastic probe with several openings with a wire electrode of stainless steel $20 \mathrm{~cm}$ in length, cross-section $0.0002 \mathrm{~m}^{2}$ in the lumen was made. The second electrode was cutaneous in the form of two standard plates. The electrodes were placed as follows: a wire electrode was placed using a nephrostome into the pelvis and ureter and was fixed to the renal capsule during operation, and its other end was led to the skin. Another electrode was placed on the skin on both sides of the 


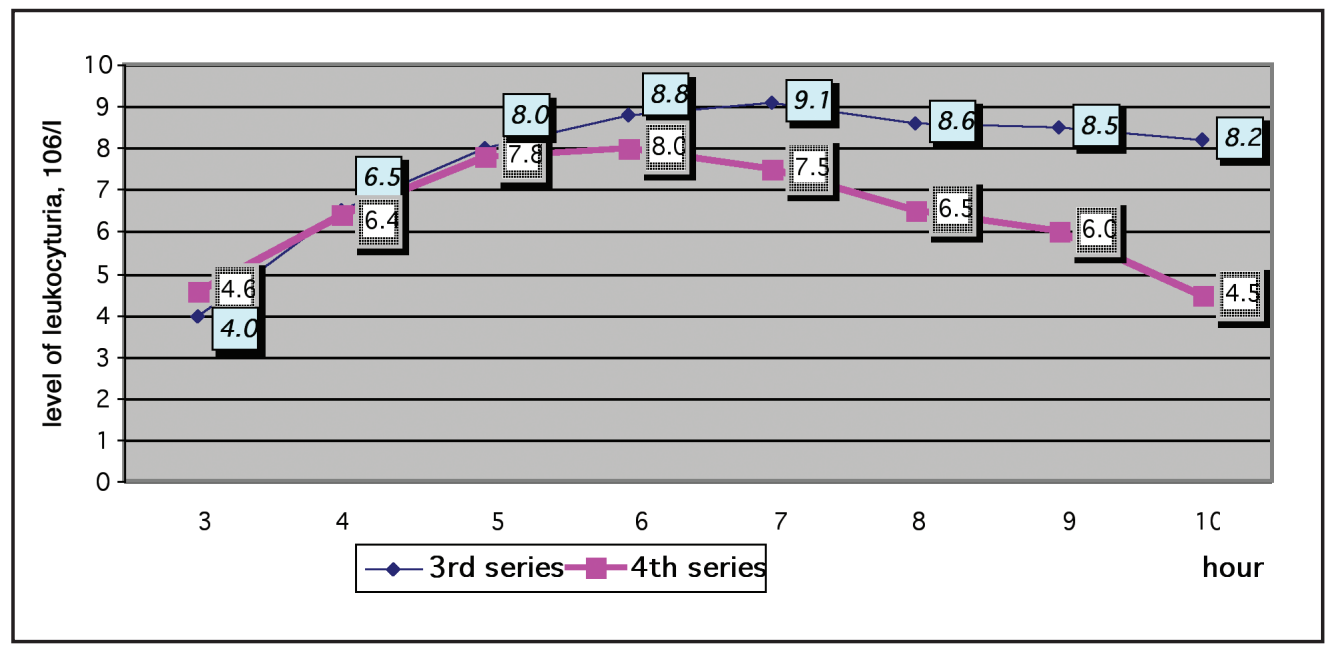

Figure 4. Dynamics of leukocyturia level in animals of the third and fourth series of experiment.

kidney projection. A Tonus device (Apparatus machine of Microwave physiotherapy, Amplipuls-5, OOO NevaTreid, St. Petersburg, Russia) designed for young children, Amplipuls-5, was used.

Ozonizing of saline solution was effected just before application using the universal medical device for ozonotherapy, Ozon UM-80 (Institute of Ozone Therapy, Kharkov, Ukraine). Administration of ozonized saline solution into the microirrigator that had been placed in the pelvis and ureter was performed by the infusion machine Ascor (Ascor SEP 21s, Poland) at the rate 5-40 mL of solution per hour within 30 minutes depending on the child's age. Sessions of DDP and ozonizing were initiated on the first post-operative day and lasted 56 days. The criteria to stop the treatment was based on significant decrease in bacteriuria and leukocyturia.

In this study the following research methods were applied: clinical, laboratory, x-ray (excretory and infusion drip urography), and echosonography (prenatal, diuretic).

Aspects such as complaints, presence or absence of fever, history of disease, and the general state of children were considered when examining the patients. Laboratory studies included clinical blood tests, levels of medium mass molecules, leukocyte toxicity index, clinical urine test, and determination the degree of bacteriuria according to Kass et al. ${ }^{5}$

Microbiological study of urine (identification, microbial count, sensitivity degree to antibacterial preparations) was carried out by the bacteriological laboratory of Regional Child Clinical Hospital No. 1 (Kharkiv).

Echosonographic studies were carried out using ultrasound device Sonolane SL-1 (Sonobane SL-1, Siemens Company, St. Petersburg, Russia) with linear and sector $3.5 \mathrm{MHz}$ transducers.

With the aim to make differential diagnoses of congenital hydronephrosis and functional pyeloectasia in newborns and children of early age, diuretic sonography according to Krasovska et $\mathrm{al}^{6}$ was performed looking into the following features: moderate hydration $(10 \mathrm{~mL} / \mathrm{kg}$ body weight) 30-40 minutes before the study, assessment of output degree of widening of the renal excretory system, determination of linear size of pelvis in 2 dimensions in transversal projection, IV introduction of $0.5 \mathrm{mg} / \mathrm{kg}$ of lasix, sonographic observation of the process 


\begin{tabular}{|lcc|}
\hline Etiology of Hydronephrosis & Absolute Number & $\%$ \\
\hline Congenital dysgenesis of PUS & 92 & 73.6 \\
Embryonic bands strangulating PUS & 6 & 4.8 \\
Aberrant vessels strangulating PUS & 11 & 8.8 \\
High exit of ureter & 9 & 7.2 \\
Deformation of ureter in PUS section & 7 & 5.6 \\
& & \\
\hline PUS=pyeloureteral segment & & \\
\hline
\end{tabular}

of filling and ejection of pelvis within 60 minutes with registration of changes of interrelation between studied values every 5 minutes with computer processing.

\section{RESULTS}

\section{Animal Experiment Results}

Stability and mobility of gentamicin in the electric field were studied in vitro. Identical spectra of absorption before and after the use of diadynamic current were obtained, which showed the preservation of the basic structure of the preparation molecule. Also, no changes were found in its antibacterial activity after the use of diadynamic currents.

Study of the histological preparations of renal parenchyma showed that 3 days after IV infection with the culture of plasmacoagulating Staphylococci the rabbits had developed acute pyelonephritis.

During the analysis of the results of the first two series of tests, it was noted that the highest concentrations of gentamicin in renal parenchyma (38.41 \pm 0.38 in the control group and $106.48 \pm 0.74$ in the main series) were found 2 hours after the beginning of the experiment, and the difference between these concentrations $(78.07 \pm 0.12$ $\mu \mathrm{g} / \mathrm{cm}^{3}$ ) was statistically significant $(P<0.001)$.

Thereafter, within 8 hours of beginning the experiment, the concentration of gentamicin in the control series was reduced by $29.17 \pm 0.31 \mu \mathrm{g} / \mathrm{cm}^{3}$, for an average hourly reduction of $3.6 \pm 0.05$ $\mu \mathrm{g} / \mathrm{cm}^{3}$, whereas in main series, reduction of concentration at 8 hours was $8.8 \pm 0.01 \mu \mathrm{g} / \mathrm{cm}^{3}$, for an hourly reduction of $1.1 \pm 0.07 \mu \mathrm{g} / \mathrm{cm}^{3}$.

The final detectable concentration of gentamicin in renal parenchyma in animals in the control group was $5.33 \pm 0.32 \mu \mathrm{g} / \mathrm{cm}^{3}$ in a 10 -hour sample of tested material. In the main series of tests, gentamicin was detected within 16 hours of observation with the final concentration of $9.38 \pm 0.09 \mu \mathrm{g} / \mathrm{cm}^{3}$ (Figure $1)$.

The results obtained showed a statistically significant difference in the level of gentamicin concentration in the kidney undergoing DDP administration of antibiotic $(P<0.01)$. There is a significant difference in the summarized determination of antibiotics in the kidney in the temporal aspect which was noted within 6 hours. A significant reduction in the concentration rate was also noted within the first 8 hours of the experiment. The antibiotic concentration reduction rate in the main series was 2.5 times less than in the control series. A significant level of probability of concentrations between the control and the main series of tests in all temporal samples $(P<0.001-0.01)$ was reached.

Using normal IV administration, gentamicin was detected in the urine after 6 hours with an increase of concentration within 2 hours by $10.83 \pm 0.08$ $\mu \mathrm{g} / \mathrm{mL}$, and then the concentration tapered until the end of observation. In 


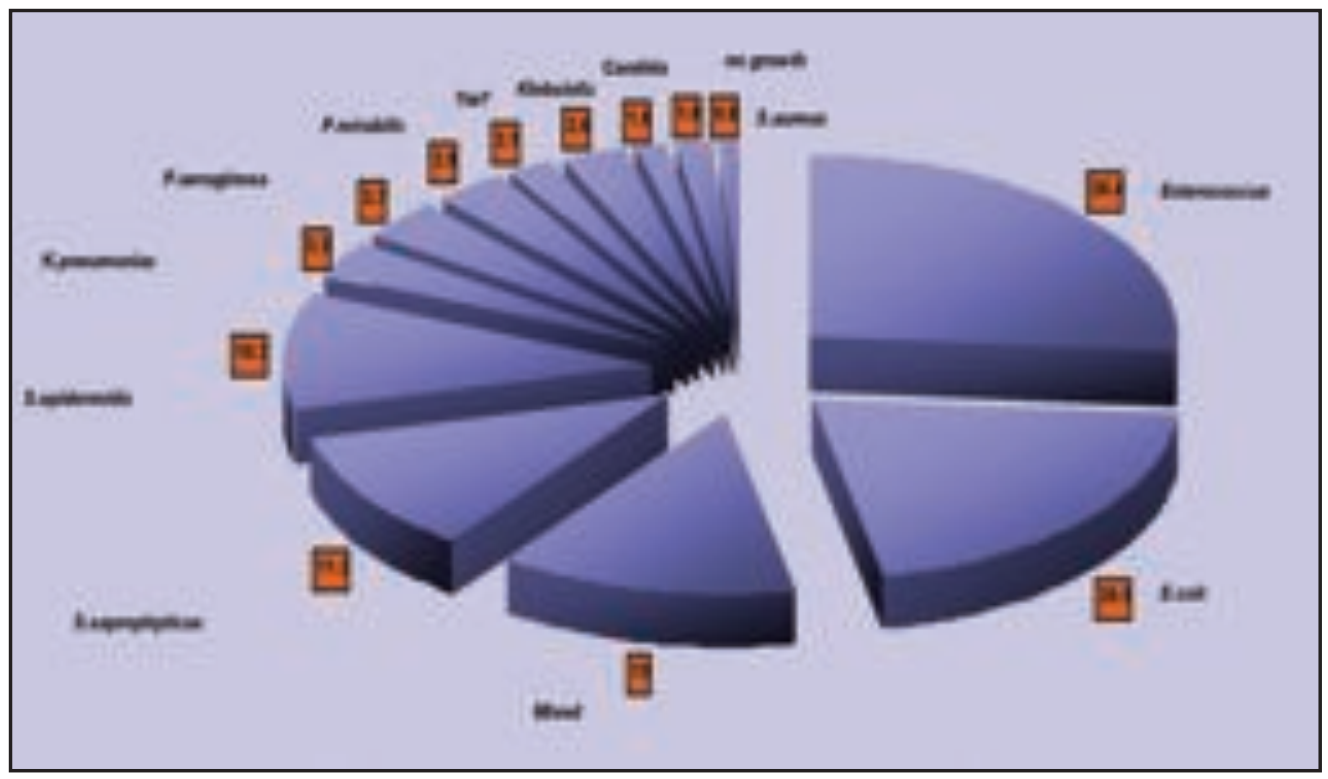

Figure 5. The content of pathogenic microflora identified in children with secondary pyelonephritis.

total, the antibiotic in the urine was found within 8 hours with an average concentration of $18.1 \pm 0.15 \mu \mathrm{g} / \mathrm{mL}$. In tests where the antibiotic was administered using DDP, it was found in the urine 4 hours after beginning the experiment with an increase of concentration within the next 6 hours. In total, antibiotic in urine was detected within 12 hours with the average concentration of $42.2 \pm 0.12 \mu \mathrm{g} / \mathrm{mL}$ (Figure 2).

Thus, average concentration of gentamicin in the urine of the main series animals was 2.3 times higher than in the controls. The time of discharging the antibiotic with the urine in the main series was 4 hours longer.

The level of bacteriuria in animals of the control series gradually reduced from the sixth day of observation, and on the tenth day it was two orders of magnitude less. In the main series reduction was detected starting from the fifth day with a four-order difference.

Beginning from the sixth day significant difference in bacteriuria level $(P<0.01)$, which progressively increased until the tenth day $(P<0.001)$, was detected
(Figure 3).

Another parameter used to determine the efficiency of treatment of pyelonephritis is leukocyturia.

Leukocyturia in both test series occurred 3 days after infection and was 4.0-4.6 x $10^{6}$. Before the fifth day in both series, leukocyturia increased equally and reached the level of 7.8-8.0 x $10^{6}$. An increase of leukocyturia in animals in the control group was found up to the seventh day $\left(9.1 \times 10^{6}\right)$. At the same time, in the test group, the reduction of leukocyturia down to $7.5 \times 10^{6}$ was detected (Figure 4).

Comparison of the obtained results demonstrates that in the main series, leukocyturia in animals was reduced from $8.0 \times 10^{6}$ to $4.5 \times 10^{6}$ on the tenth day of observation, and in the control series it was reduced to $8.2 \times 10^{6}$.

\section{Clinical Study Results}

Statistical analysis showed that in $73.6 \%$ of the cases, hydronephrosis was caused by congenital dysgenesis of the ureteric muscular layer in the area of the pyeloureteral segment (PUS). In other 
Table 2. Incidence and severity of secondary pyelonephritis depending on the stage of hydronephrosis

\begin{tabular}{|c|c|c|c|c|c|c|c|}
\hline \multirow[b]{2}{*}{$\begin{array}{l}\text { State of } \\
\text { Hydronephrosis }\end{array}$} & \multirow[b]{2}{*}{ Number } & \multirow[b]{2}{*}{$\%$} & \multicolumn{2}{|c|}{$\begin{array}{c}\text { Secondary } \\
\text { Pyelonephritis }\end{array}$} & \multicolumn{3}{|c|}{ Leukocyturia } \\
\hline & & & $\begin{array}{c}\text { Absolute } \\
\text { number }\end{array}$ & $\%$ & Insignificant & Moderate & Pyuria \\
\hline \multicolumn{8}{|l|}{ Control group $(n=50)$} \\
\hline Stage I - initial & 16 & $32 \pm 7$ & 7 & $44 \pm 13$ & $3(43 \pm 20)$ & $4(57 \pm 20)$ & - \\
\hline Stage II - marked & 25 & $50 \pm 7$ & 15 & $60 \pm 10$ & $2(13 \pm 9)$ & $8(53 \pm 13)$ & $5(33 \pm 13)$ \\
\hline Stage III - terminal & 9 & $18 \pm 5$ & 9 & 100 & - & $2(22 \pm 15)$ & $7(78 \pm 15)$ \\
\hline Total & 50 & 100 & 31 & $62 \pm 12$ & $5(16 \pm 7)$ & $14(45 \pm 9)$ & $12(39 \pm 9)$ \\
\hline \multicolumn{8}{|l|}{ Main group $(n=75)$} \\
\hline Stage I - initial & 32 & $43 \pm 6$ & 14 & $44 \pm 9$ & $6(43 \pm 14)$ & $8(57 \pm 14)$ & - \\
\hline Stage II - marked & 37 & $49 \pm 6$ & 20 & $54 \pm 8$ & $5(25 \pm 10)$ & $11(55 \pm 11)$ & $4(20 \pm 9)$ \\
\hline Stage III - terminal & 6 & $8 \pm 3$ & 6 & 100 & - & $1(17 \pm 17)$ & $5(83 \pm 17)$ \\
\hline Total & 75 & 100 & 40 & $53 \pm 8$ & $11(28 \pm 7)$ & $20(50 \pm 8)$ & $9(22 \pm 7)$ \\
\hline
\end{tabular}

children, hydronephrosis was caused by aberrant vessel $(8.8 \%)$, high exit of the ureter $(7.2 \%)$, ureter angulation $(5.6 \%)$, or embryonic bands (4.8\%) (Table 1).

Secondary pyelonephritis complicated the course of hydronephrosis in an average of $56.8 \%$ of the observations. The incidence of pyelonephritis in children of various age groups was different. Children in the first year of life showed an incidence of $33 \pm 8 \%$, and in children in the first half-year period it was $18 \pm$ $10 \%$. At the age of 1 through 5 years, pyelonephritis was found in $45 \pm 11 \%$ of the observations, and in children above 10 years, it was found in $96 \pm 4 \%$.

We studied the microbiological structure of pyelonephritis (121 urine inoculations). The dominating microorganism was Enterococcus (26.4\%). Other microorganisms present were E. coli (20.5\%), S. saprophyticus (11.2\%), and S. epidermidis $(10.3 \%)$. Other microorganisms were found much less often (Figure 5).

Analysis of microflora sensitivity to antibiotics found that almost all pathogens showed low sensitivity to antibiotics of penicillin group, and were rather sensitive to gentamicin and cephalosporins. Thus, the isolated microflora displayed high resistance to antibiotics. In more than half of the studied antibiotics, sensitivity did not exceed $50 \%$. The most efficient antibiotics were imipinem, $80.3 \%$ sensitivity; ceftazadime, $83.4 \%$ sensitivity; cefotaxime, $86.9 \%$ sensitivity; and aztreonam, $89.3 \%$ sensitivity.

Analyzing the results of the treatment of children in both groups, it is worth noting that secondary pyelonephritis is the most frequent and constant complication of congenital hydronephrosis in children. Pyelonephritis incidence sharply differs in various age categories. Thus, in children above 10 years of age pyelonephritis was present in nearly all (96\%) of the observations; in children below 6 months of age pyelonephritis was found only in $18 \%$. A distinct dependence of secondary pyelonephritis incidence from the stage of hydronephrotic transformation of kidney is evident. The first-stage hydronephrosis was accompanied by pyelonephritis in $44 \pm 15 \%$ of the observations, and in the third-stage, hydronephrosis was complicated with pyelonephritis in $100 \%$ of the observa- 


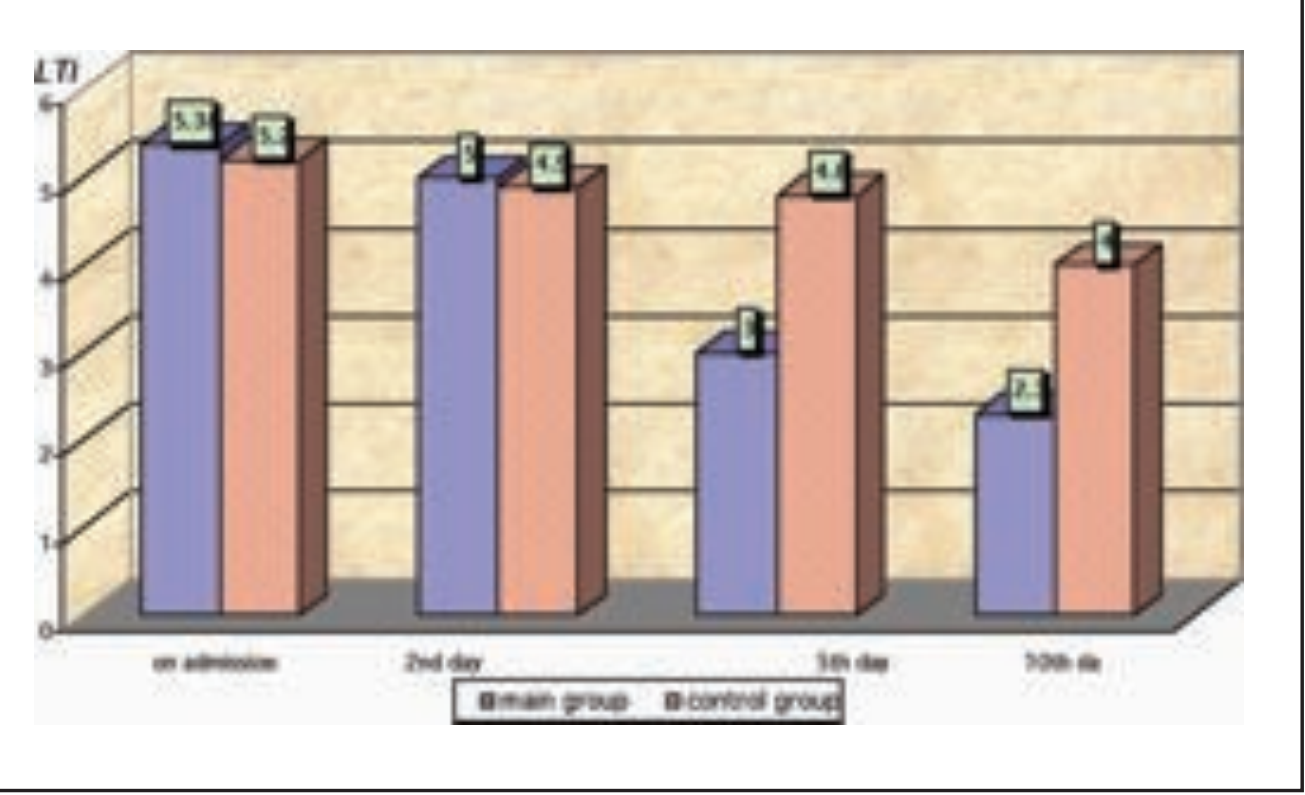

Figure 6. Level of leukocyte toxicity index (LTI) in children with secondary pyelonephritis.

tions (Table 2).

Comparing the two groups of observations, it is necessary to emphasize that in the main group, the number of children under the age of 1 year was considerably higher when compared with the control group $(14 \pm 5 \%)$. In the main group, children aged 6 months and younger make up $61 \pm 9 \%$ of the children under the age of 1 , and in the control group there were no children under the age of 6 months.

Overall, the comparative analysis of the clinical and laboratory findings for children in both groups showed that the disease dynamics in children of the main group were better than those of the control group.

Analysis of laboratory findings showed that leukocytosis in children of the main group significantly reduced from the second day, and in the controls from the tenth day. The dynamics of the white blood cell (WBC) formula was the same. The erythrocyte sedimentation rate (ESR) in the control group showed no changes before the tenth day, and in the main group demonstrated significant changes on the fifth day of observation. The level of medium-mass molecules in children of the main group differed significantly from the initial level on the second day, and in the control group, this difference in this parameter did not reach statistical significance until the tenth day.

Leukocyte toxicity index (LTI) in children of the main group was significantly reduced on the fifth day, and in the control group at that time it showed no considerable changes (Figure 6). Analysis of bacteriuria dynamics in children ages 1-5 in the main group showed that after the first session of pelvis and ureter treatment with ozonized saline solution, the level fell considerably, almost down to the normal level from an initially high parameter (up to $10^{8}$ ). Only on the second and third day the level of bacteriuria partially recovered to $10^{8}-10^{6}$, although in the prevailing majority of observations it never exceeded $10^{5}-10^{3}$. This shows efficiency of the administered therapy. In the majority of cases, the urine was sterile by the fourth or fifth day (Figure 7). 


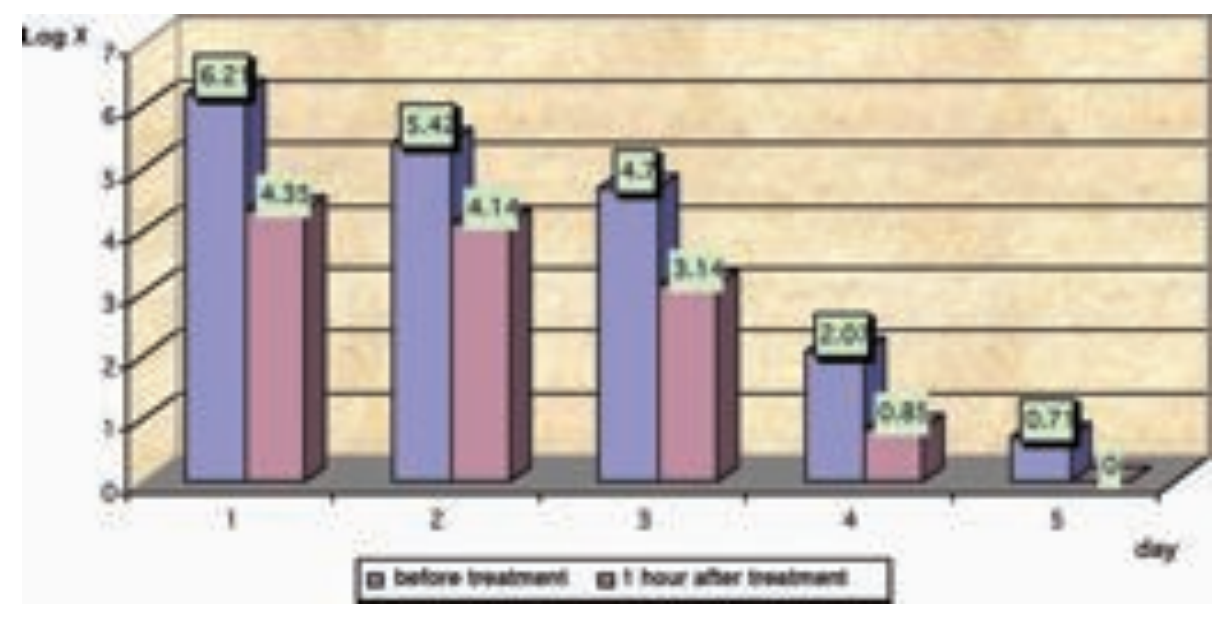

Figure 7. Dynamics of general microbial contamination of the urine in children of main group (15 years old) when performing treatment of the pelvis and ureter with ozonized saline solution.

In older children, urine sanitation was much slower than in younger children. This may be due to the increased resistance of microflora. Thus, after the first sanitation, no considerable changes of bacteriuria level were detected, but on the second day after the second sanitation, in several children the parameter was normal, although initial bacteriuria on the third day slightly increased. Efficient reduction of bacteriuria level in these children was found after the fourth or fifth sanitation $(P<0.001)$.

All children in the main group underwent organ-preserving plastic operations, and in the control group the researchers had to perform nephrectomy in $3(6 \pm 3.4 \%)$ patients.

According to the histological studies conducted using the original technique, antibacterial therapy limited the area of spreading infection to the cortical layer of the renal tissue. Moderate intensity and the spread of inflammatory infiltration of the tissue, an almost complete absence of leukocytic component of cellular reaction, and an early termination of the inflammatory process in the kidneys resulted in full restitution of the tissue.

\section{DISCUSSION}

Methods of combined application of intratissue DDP of antibiotics and ozonized solutions in treating secondary pyelonephritis, complicating congenital hydronephrosis in children was worked out. These methods significantly improved immediate and late results of therapy.

Recommendations regarding usage of intratissue DDP of antibiotics and ozonized solutions in the therapy of secondary pyelonephritis in children, based on research results, have been implemented in practice in the surgical clinic of Kharkiv Regional Child Clinical Hospital No. 1, Kremenchuk Child Hospital and Regional Child Hospital in Sumy. ${ }^{7}$

Our experimental studies showed that antibacterial therapy of pyelonephritis in animals, treated using intratissue diadynamophoresis of antibiotics, considerably differs in efficiency from the standard protocol of antibiotic therapy. Early elimination of the inflammatory process in the kidneys facilitates minimum morphological changes in the parenchyma and rapid renovation of all structural and functional impairments. 
The positive experimental results enabled us to introduce our methods of antibacterial therapy into clinical practice.

Pyeloureteroplasty in hydronephrosis in children of an early age is a microsurgical procedure that can be performed only with optical magnification and use of microsurgical equipment. Therefore, we developed and introduced into practice the technique of video-assistance in which a laparoscope tubus with video camera is introduced into the surgical wound. The operative area is projected on the monitor, enabling us to magnify considerably the anatomic structure subjected to anastomosis and providing an adequate view of the operation field for the surgeon and assistants.

We performed 10 operations with video-assistance for newborn children and two 3-month-old children. The earliest successful operation was for a 3week-old child with bilateral hydronephrosis. The second kidney was operated on when the child was 1.5 months old.

While studying the results at each 3month period following the initiation of the study it was found that during the first year of observation, relapses of pyelonephritis in hydronephrosis of third-degree of severity were recorded in both groups, but during the second year a significant difference was detected. In the control group, relapses of pyelonephritis were recorded in $67 \pm$ $17 \%$ of children, and in the main group, only in $33 \pm 21 \%$ of patients. After 3 years of observation, in the main group relapses were recorded in $17 \pm 17 \%$ of children, and in the control group, in 44 $\pm 18 \%$ of patients.

Thus, analysis of the results of our experimental clinical research enables us to state that secondary pyelonephritis constantly accompanies congenital hydronephrosis in children. It consider- ably complicates the course of this disease and worsens treatment results.

Early prenatal diagnosis of the malformations of urinary system and highlevel postnatal dynamic observation enable us to diagnose timely congenital hydronephrosis and to perform early corrective operations before the development of pyelonephritis. This can considerably improve the short-term and long-term results of treatment.

Therapy of secondary pyelonephritis is a complex clinical problem stipulated with high resistance of contemporary microflora and nephrosclerotic changes resulting from hydronephrosis and impairment of microcirculation in the kidney. ${ }^{8}$

With the aim to improve efficiency of antibacterial therapy when treating secondary pyelonephritis at all therapy stages, it is possible to successfully use the method of intratissue diadynamopherosis of antibiotics, which apart from its principal application - formation of high and continuous concentrations of antibacterial agents in inflammation foci-creates favorable conditions for the course of reparative processes in the operated organ area, and performs electric stimulation of the pelvis and ureter, and thus facilitates adequate urodynamics. ${ }^{9}$

For direct sanitation of the urinary tract and urine it is possible to effectively use drop administration of ozonized saline solution into the pelvis and ureter. This technique does not require large volumes of antiseptic and long-term installations. This is very important in the conditions of small volumes of pelvis and ureter in children.

Complex application of the developed scheme of therapy of congenital hydronephritis, complicated with pyelonephritis, makes it possible to considerably improve results of therapy and to avoid organ-removing operations in children. ${ }^{10-13}$ 


\section{CONCLUSION}

Secondary pyelonephritis is the most frequent complication of congenital hydronephrosis in children. The incidence of secondary pyelonephritis depends on the child's age: in children under six months, it is $18 \pm 10 \%$, and in children above 10 years old the incidence is $96 \pm 4 \%$. Microflora causing secondary pyelonephritis is diverse. The main factor is coccus flora $(24.47 \%)$ in association with $E$ coli (22.7). More than half of the studied antibiotics show microflora sensitivity not exceeding $50 \%$. High sensitivity was demonstrated by imipinem, ceftazadime, cefotaxime, and aztreonam.

Application of intratissue diadynamopherosis of antibiotics enables us to increase the concentration of antibiotic in renal parenchyma by eight times and in urine by 3.6 times in comparison with standard antibiotic therapy, to create its continuous concentration during 16 hours, and to carry out early electrostimulation of pelvis and ureter facilitating quicker healing the anastomosis and early recovery of urodynamics. ${ }^{14}$ The original technique of antibacterial therapy limited the spread of inflammation affection in the kidney only in the cortical layer. Moderate intensity and the spread of inflammatory infiltration of tissues, an almost complete absence of leukocyte component of cellular reaction and an early elimination of inflammatory process stipulating complete restitution of renal tissues were noted.

Application of ozonized saline solution with ozone concentration of 6-8 $\mathrm{mg} / \mathrm{L}$ for washing the renal excretory system enables us to perform full and stable sanitation of urine, to increase oxygenation of anastomosis area (facilitating early healing) within 4-5 days. ${ }^{15}$ Early surgical correction of congenital hydronephrosis in children and intensive conservative therapy of secondary pyelonephritis enabled us to create favorable conditions for performing organ-preserving operations, gave early recovery of functional renal impairments, produced uncomplicated course of post-operative period, and reduced the incidence of pyelonephritis from $30.8 \%$ down to $19.6 \%$.

For early diagnosis of congenital hydronephrosis in children and timely treatment of this disease it is necessary to apply prenatal ultrasound diagnosis and postnatal dynamic observation and comprehensive examination. For direct sanitation of renal excretory system and urine it is possible to effectively use instillations of ozonized saline solution into pelvis and ureter with ozone concentration of $6-8 \mathrm{mg} / \mathrm{L}$ daily within 5-6 days with exposition up to 30 minutes. For treating the congenital hydronephrosis it is recommended to perform early surgical correction which facilitates the recovery of adequate urodynamics of upper urinary tract and prevents development of nephrosclerotic and microcirculatory changes in the kidneys and secondary pyelonephritis. This favorably affects short- and long-term functional results.

Taking into account the diversity of microflora causing secondary pyelonephritis and its manifested resistance to antibacterial agents, it is required to use highly efficient antibiotics of wide spectrum of action such as imipinem, ceftazadime, cefotaxime, and aztreonam. With the aim to improve efficiency of antibacterial therapy it is recommended to apply intratissue diadynamopherosis of antibiotics under the original technique described in this research. This enables us to form high and continuous concentrations of antibacterial agents in renal tissues and urine with simultaneous electric stimulation of pelvis and ureter in the early post-operative period. The research has solved the urgent problems of improving results of treating the congenital 
hydronephrosis complicated with pyelonephritis in children with early surgical correction of malformation and improvement of conservative methods of therapy.

\section{REFERENCES}

1. Lapshin VV, Davydenko VB, Vyun VV, et al. Prophylaxis and treatment of postnatal pyelonephritis at surgical correction of hydronephrosis in children. Medical Practice. 2001;2:91-93.

2. Tsyganenko AY, Davydenko VB, Vasylchenko VN, et al. Development of efficient methods of treating the purulent septic processes in children on the basis of integrated clinical and microbiological studies. Exper Clin Med. 2002;3:73-75.

3. Yousef MA. Experimental substantiation of diadynamopherotic antibacterial therapy of pyelonephritis. Exper Clin Med. 2002;2:119121.

4. Yousef MA. Contemporary approaches to treating the secondary pyelonephritis in children. Today Tomorrow Med. 2002;4:87-89.

5. Kass EH, Svanborg-Eden C. Host-parasite interactions in urinary tract infections. University of Chicago Press, Chicago, 1989, 194-197.

6. Krasovska IV, Rostovskaya VV, Babanin IL. Diagnostic sonography for obstructive disorders and urodynamics of the upper urinary system in hydronephrosis, Pedtr Surg. 2002;2:21-26.

7. Yousef MA, Davydenko VB. New method of treating secondary pyelonephritis in hydronephrosis in children. Dig Research Kyiv Med Acad Postgrad Ed, Kyiv. 2001:314315 .

8. Yousef MA, Davydenko VB. Method of antibacterial therapy at treating pyelonephritis in children. Ped Obstet Gynecol. 2003;3:69.
9. Lapshin VV, Yousef MA, Davydenko VB. Declaration Patent of Ukraine for Invention No. 52255A, MPK 7 A61P13/00. The method of sanitation of urinary system at secondary pyelonephritis. Kharkiv State Medical University, Application No. 2002032497 dated 29 March 2002; Published: December 16, 2002. Bulletin No. 12.

10. Lapshin VV, Davydenko VB, Vyun VV, et al. Optimization of post-operative therapy for pyelonephritis in hydronephrosis in children. Surgery 2001. Achievements in contemporary surgery: Materials of III ${ }^{r d}$ Russian Scientific Forum. Moscow. 2001:206-207.

11. Lapshin VV, Davydenko VB, Yousef MA. Facilitation of therapy of post-operation pyelonephritis in children. Proceedings of VI Conference of Pediatric Urologists of Ukraine 25-26 October 2001: Theses of Reports. Zhytomyr. 2001:24-25.

12. Davydenko VB, Yousef MA. Improvement of efficiency of antibacterial therapy of secondary pyelonephritis in children. Materials of 20th Congress of Surgeons of Ukraine. Ternopil. 2002:311.

13. Shtyker SY, Yousef MA, Davydenko VB. New trends in ozone therapy in pediatric surgery. New techniques of recovery using natural and performed factors (physiotherapy, ozone therapy, resorts). Materials of Research and Practical Conference. Kharkiv. 2002:194-197.

14. Yousef MA. Methods of improving the antibacterial therapy of pyelonephritis in children. 7th International Medical Congress of Students and Young Scientists: Proceedings of Congress. Ternopil. 2003:62.

15. Shtyker SY, Yousef MA, Davydenko VB. Prospects of applying ozone in pediatric surgery. Third International Research and Practical Conference "Science and Social Problems of Society: Medicine, Pharmacy, Biotechnology": Proceedings of the conference. Kharkiv. 2003:237. 